\title{
Study on heat sources of horizontal lathe machine by using inverse method
}

\author{
Van-The Than****, Chi-Chang Wang**, Ming-Lun Chang** and Thi-Thao Ngo* \\ * Faculty of Mechanical Engineering, Hung Yen University of Technology and Education, Khoai Chau District, Hung Yen Province, \\ Vietnam \\ ** Department of Mechanical and Computer-Adided Engineering, Feng Chia University, No. 100 Wenhwa Rd., Seatwen., Taichung, Taiwan \\ 40724, R.O.C \\ *,***Corresponding Author: thanthe.ck@gmail.com
}

Submitted :04/02/2020

Revised : 24/12/2020

Accepted :09/01/2021

\begin{abstract}
Thermal has a significant effect on high speed machine tools. When temperature grows up, it often causes processing errors and further reduces product quality. This paper aims to investigate magnitude of heat sources and temperature distribution in a horizontal lathe based on inverse method. In the present thermal model, there is not only consideration of spindle, but also analyzing relative structures. Simulated and experimental temperatures are used as input data to predict heat sources and temperature field. Effects of speed, number measured points, measurement errors, and measured distance on predicted results are analyzed. Results indicate that this inverse method can accurately estimate the heat sources based on two measured temperatures at front and rear outer rings. The trend of estimated heat sources is then compared to measured load rate. Results herein are useful information for designing horizontal lathe spindle and reducing thermal errors.
\end{abstract}

Keywords: Inverse heat conduction problem; Conjugate gradient method; Finite element analysis; Spindle; Heat source.

\section{INTRODUCTION}

A spindle is an important part, which significantly affects machining processes and a key component with the largest temperature change in machine tool. There are parameters such as spindle speed, cutting force, belt pull, ball type, lubricating oil, and preload that are important factors for spindle design; and they can be used to evaluate magnitude of spindle bearing heat source. However, it is difficult to directly measure spindle bearing heat sources. Therefore, this study applies inverse heat transfer method to obtain the magnitude of heat source in rotating bearing based on measured temperature data. For spindle thermal behavior, Bossmanns, B. and J.F. Tu (Bossmanns and Tu, 1999) used finite difference method to simplify a spindle model and obtained temperature distribution in rotation state. Holkup et al. (Holkup et al., 2010) proposed combining finite element method with heat resistance model to build a spindle mechanism model and analyze temperature rise thermal behavior. As requirement machining accuracy and interest of thermal compensation grow up, Yang et al. (Yang et al., 2018) used finite element method to consider spindle temperature rise distribution and thermal elongation; and they further employed second-order regression model to obtain a relation between temperature and thermal elongation. Convection coefficient has a great effect on 
spindle thermal model, and it will be significantly changed with changing spindle speed. Lequien et al. (Lequien et al., 2018) used hybrid modeling to obtain convection coefficient of spindle under different rotation and environmental conditions. Bearing is a main heat source of spindle mechanism, and the bearing is the most vulnerable component over another. In order to increase spindle thermal analysis accuracy and avoid excessive temperature rise, Jedrzejewski et al. (Jedrzejewski and Kwasny, 2010) based on the ball size, preload, and kinematic viscosity coefficient of lubricating oil calculated the magnitude of heat source of roller and ball bearings.

Most of the above studies used academic and theoretical derivation to set parameters for the model under spindle operating condition and then obtained temperature rise distribution or thermal elongation, i.e., solving the direct problem. However, if bearing heat source, which is difficult to be directly measured, cannot be found, this would cause large errors as a result. Therefore, the bearing heat source of spindle is obtained by inversed method; hence, magnitude of bearing preload can be determined under different cutting speeds. Inverse Heat Conduction Problem (IHCP) started from Shumakov (Shumakov, 1957) and Stolz et al. (Stolz Jr, 1960) using inverse method to analyze heat transfer problem of missile nozzles and rocket nozzles. Stolz (Stolz Jr, 1960) used Superposition Principle for numerically inverse method of the heat transfer rate during quenching of objects in simple profiles. In these studies, if the time interval is short, the values are unstable, and the measurement errors or delay phenomenon turns the IHCP into Ill-Posed Problem. Beck (Beck, 1968) used convolutional values for inversion and used least squares method to obtain a heat flux of body surface. The comparison of this study and Stolz indicates that estimated result is more stable and reduced about 5/6 of calculation steps. Beck (Beck, 1970) used finite difference and proposed the Future Temperature concept for analyzing body surface temperature and heat flux density, and the numerical instability from short time interval in previous studies was improved. Recently, inverse problem is mostly combined with Optimal Theory to calculate complex model parameters. Huang et al. (Huang and Lo, 2006) used the Steepest Descent Method (SDM) to compute the spindle heat flux for subsequent thermal design for spindle water jacket. However, the iterative computation efficiency in SDM decreases greatly when approaching an optimal solution. Wang et al. (Wang et al., 2014) used Newton-Rasphon Method to compute the radial and axial forces as well as angular displacement of highspeed spindle bearing. Solving process of Newton's method, the Hessian Matrix has a large amount of computation and actual measurement error leading to huge deviation of computing result. Huang et al. (Huang et al., 2016) used Conjugate Gradient Method and two-dimensional axisymmetric model to analyze the spindle and obtained timedependent heat flux of front and rear bearings. To sum up previously mentioned review, this paper will simplify a three-dimensional $\mathrm{CNC}$ lathe machine model and use conjugate gradient method to compute the time-dependent spindle bearing heat source and discuss its relationship to dynamic preload.

\section{INVERSE HEAT TRANSFER MODEL}

\section{Thermal Model}

As overall structure of $\mathrm{CNC}$ horizontal machine tool lathe machine is very huge and complex, direct finite element analysis may result in too large amount of calculation, consuming time and the grid error may be more severe. Figure 1 shows a section built under the spindle and Figure 2 presents boundary conditions of section, which are set according to measured temperature. The spindle is separated from the other mechanisms of lathe machine to reduce amount of analytical calculation. In addition, because of the complex and asymmetric machine tool, a threedimensional transient heat transfer model is used for analysis in this paper. The energy conservation law can be expressed as follows:

$\Delta E_{\text {sys }}=E_{\text {in }}-E_{\text {out }}$ 
Any system must satisfy Eq. (1), and time variation of intersystem energy, energy transferred in and out of the system, and heat generation rate per unit volume of system are compiled as follows:

$\rho c \frac{\partial T}{\partial t}=\nabla(k \nabla T)+q$

where $T$ and $t$ are the spindle temperature and time, respectively; $k, \rho$ and $c$ are the thermal conductivity (44.5 $W / \mathrm{m} \cdot \mathrm{K})$, density $\left(7850 \mathrm{~kg} / \mathrm{m}^{3}\right)$, and specific heat $(475 \mathrm{~J} / \mathrm{kg} \cdot \mathrm{K})$, respectively; because of non-large temperature rise of machine tool, the specific heat and thermal conductivity are assumed to be constant. $\dot{q}$ is heat source of the rotating spindle bearing; this physical quantity will be obtained by using inverse method in next section.

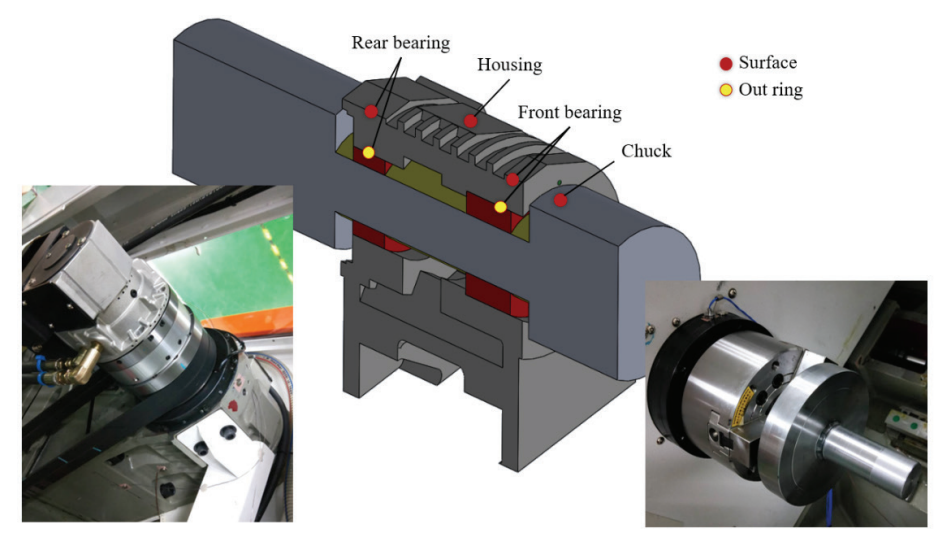

Figure 1. A simplified model and positions of measurement points.

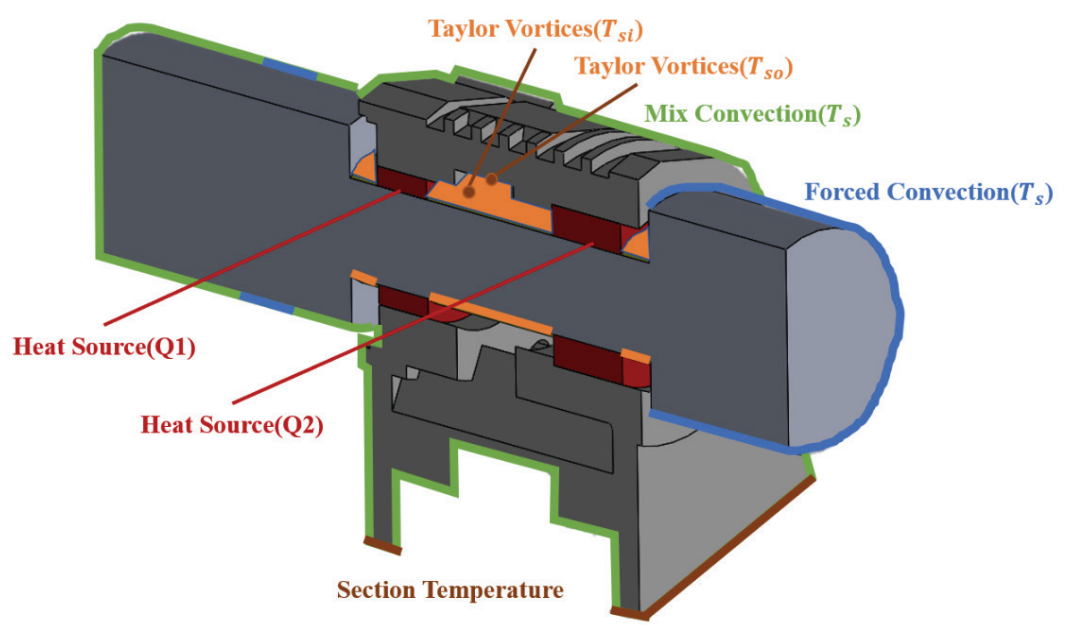

Figure 2. Boundary condition of thermal model.

As shown in Figure 2, temperature field in Eq. (2) shall be computed with initial and boundary conditions, set as follows:

$T=T_{\infty}$ at $\mathrm{t}=0$ 
$-k \frac{\partial T}{\partial r}=h_{f o}\left(T_{s}-T_{\infty}\right)+\varepsilon \varsigma\left(T_{s}^{4}-T_{\infty}^{4}\right)$ for force convection

$-k \frac{\partial T}{\partial r}=h_{n a}\left(T_{s}-T_{\infty}\right)+\varepsilon \varsigma\left(T_{s}^{4}-T_{\infty}^{4}\right)$ for mix convection

$-k \frac{\partial T}{\partial r}=h_{T a}\left(T_{s i}-T_{s o}\right)+\varepsilon \varsigma\left(T_{s i}^{4}-T_{\infty}^{4}\right)+\varepsilon \varsigma\left(T_{s o}^{4}-T_{\infty}^{4}\right)$ for Taylor convection

where the temperature subscripts s, si, so and $\infty$ represent the spindle outer ring surface, spindle arbor surface, spindle outer ring inner surface, and ambient temperature, respectively; $\varepsilon$ and $\varsigma$ are the body surface emissivity (Steel AISI4340 set as 0.2) and Stefan-Boltzmann constant (5.67E-8 $\left.\mathrm{W} / \mathrm{m}^{2} \cdot \mathrm{K}^{4}\right) ; h_{f o}, h_{n a}$ and $h_{T a}$ are heat convection coefficients of forced, natural, and Taylor vortices, respectively. A relationship between heat convection coefficient and average Nusselt number is expressed as follows:

$h=\frac{N u k_{\text {air }}}{D}$

where $k_{\text {air }}$ and $D$ are air thermal conductivity and characteristic length, respectively.

As shown in

Figure 2, the spindle chuck mechanism and rear belt pulley are rotating components of spindle, which are exposed to the environment; the surface is set as forced convection. An empirical equation (Kendoush, 1996) of average Nusselt number of rotating cylinder is approximated by

$$
N u_{f o}=0.5 \int_{0}^{2 \pi} N u_{\theta} d \theta=0.6366(\operatorname{RePr})^{0.5}
$$

For the natural convection, the average Nusselt number of long horizontal cylinder can be determined as (Churchill and Chu, 1975)

$$
N u_{n a}=0.6+\frac{0.38 R a_{D}^{1 / 6}}{\left[1+(0.559 / \mathrm{Pr})^{9 / 16}\right]^{8 / 27}}
$$

According to (Childs and Long, 1996), the average Nusselt number of Taylor vortex flow in the gap of long horizontal concentric cylinders is estimated by

$$
N u_{T a}=0.409+\left(\frac{T a^{2}}{f}\right)^{0.241} \text { for } 10^{4}<\frac{T a^{2}}{f}<10^{7}
$$


where $T a$ and $f$ are Taylor number and geometric parameter, respectively.

Solving governing equations directly to find the distributed temperature is complicated and time consuming. Therefore, in this study, COMSOL Multiphysics software is used to construct and solve thermal model for the lathe spindle. All heat transfer coefficients such as convection and radiation are calculated and are then key in the software. After that, the temperature distribution in all spindle will be obtained. This is considered as a direct problem in the inverse heat transfer process.

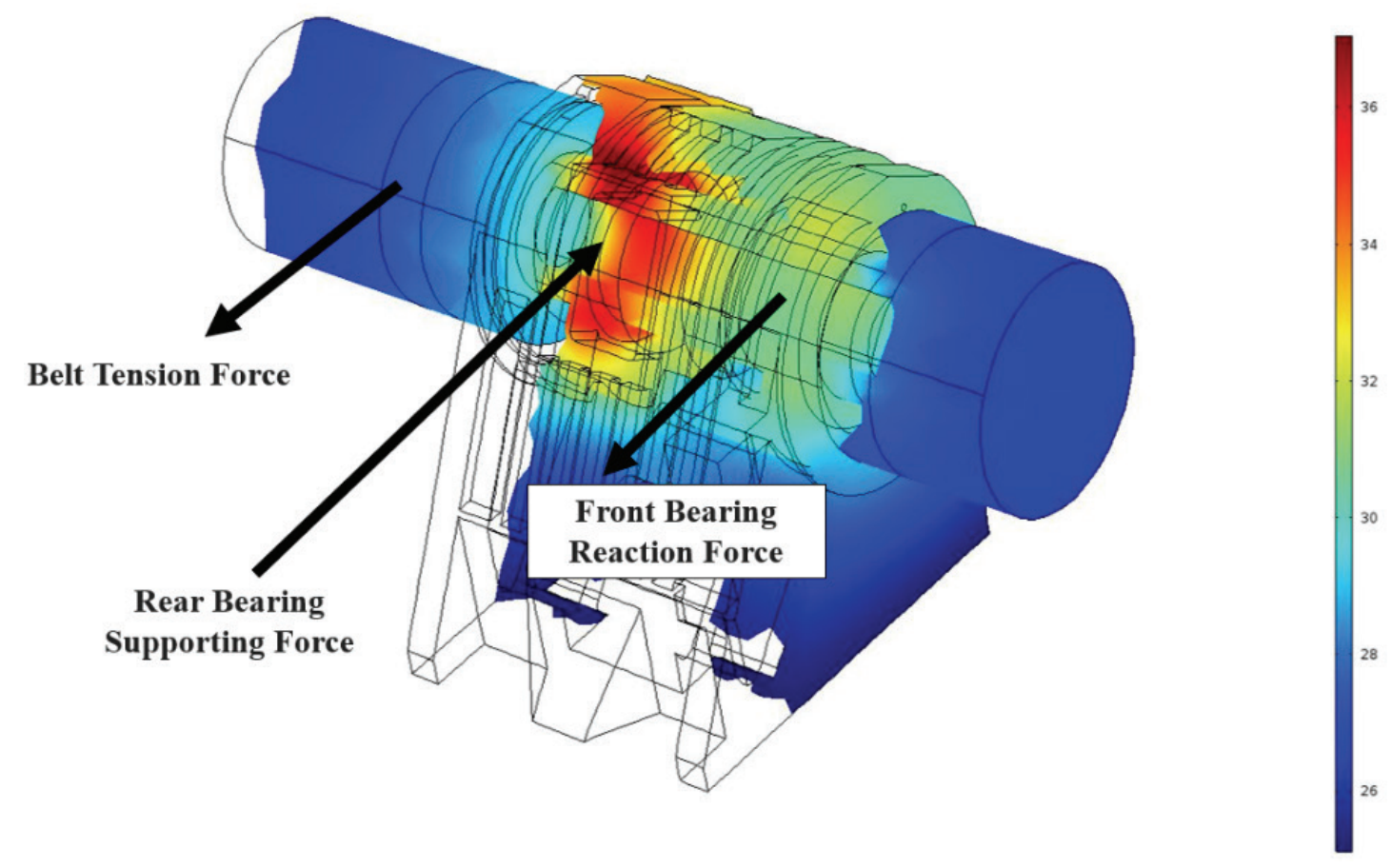

Figure 3. Temperature and force distribution by running for 2 hours in 4000RPM.

\section{Inverse Method}

An inverse method is a process of computing an optimal value, thus optimizing search efficiency becomes an important arithmetic skill. This paper uses conjugate gradient method to compute two heat sources in the front and rear bearings of machine tool spindle. By this method, small calculation of secondary termination characteristic and only first derivation must be spent while the inverse heat source is still performed more efficiently and accurately.

In order to solve heat inverse problem, unknown physical quantities and objective function must be defined first. Heat source due to friction in bearing is assigned as unknown quantity. As shown in Figure 1 and 3, the horizontal lathe spindle rotating mechanism is driven by motor through belt; and the driven positions are not symmetric. Hence, the front and rear bearings receive different normal forces and further different heat sources are generated. Therefore, the front and rear bearings are regarded as two independent unknown and time-dependent heat sources $\left(Q_{1}\right.$ and $\left.Q_{2}\right)$, represented by a vector as follows:

$$
Q=\left[Q_{1} Q_{2}\right] \text { at } t=t_{n}, t_{n}=n \Delta t, n=1,2,3 \ldots
$$


In order to discretize time, the time is divided by index $n$ into several small intervals, and each interval is $\Delta t$. As for objective function, this study uses the least square error

$$
\left.J_{n}=\sum_{i=1}^{Z}\left[T_{i n v}\left(r_{i}, \theta_{i}, z_{i}, t_{n+1}\right)\right)-\left[T_{m}\left(r_{i}, \theta_{i}, z_{i}, t_{n+1}\right)\right)\right]^{2}
$$

An objective function is minimized for finding unknown heat source; in which $T_{i n v}$ and $T_{m}$ are inverse and measured temperature respectively; and $N$ is the total number of measuring points. To minimum the object function, the following iterative is used:

$$
Q_{n}^{(k+1)}=Q_{n}^{(k)}-\beta_{n}^{(k)} P_{n}^{(k+1)}
$$

A conjugate vector $\vec{P}$ provides the function correction direction, and an optimal step $\beta$ is calculated to obtain time-dependent heat source of two bearings by the most efficient numerical iteration. In order to compute the optimal unknown physical quantities, the conjugate gradient direction and step size must be obtained first for inverse problem. Conjugate gradient direction $\overrightarrow{\mathrm{P}}$ is expressed by

$P_{n}^{(k+1)}=\nabla J_{n}^{(k)}+r_{n}^{(k)} P_{n}^{(k)}$

An objective function gradient, $\nabla J_{n}^{(k)}$, is computed by Central Finite Different Method.

$$
\nabla J_{n}^{(k)}=\left[\frac{J_{n}\left(Q_{1}^{(k)}+\Delta Q, Q_{2}^{(k)}\right)-J_{n}\left(Q_{1}^{(k)}-\Delta Q, Q_{2}^{(k)}\right)}{2 \Delta Q} \frac{J_{n}\left(Q_{1}^{(k)}, Q_{2}^{(k)}+\Delta Q\right)-J_{n}\left(Q_{1}^{(k)}, Q_{2}^{(k)}-\Delta Q\right)}{2 \Delta Q}\right]
$$

A conjugate factor, $r$, is determined by

$$
r_{n}^{k}=\frac{\left\|\nabla J_{n}\left(Q_{n}^{(k+1)}\right)\right\|^{2}}{\left\|\nabla J_{n}\left(Q_{n}^{(k)}\right)\right\|^{2}}
$$

An optimal step function, $\beta$, is obtained as

$$
\beta_{n}^{(k)} \frac{\Delta Q \sum_{i=1}^{M}\left(T\left(Q_{n}^{(k)}\right)-T_{m}\right)\left[T\left(Q_{n}^{(k)}+\Delta Q P_{n}^{(k)}\right)-T\left(Q_{n}^{(k)}\right)\right]}{\sum_{i=1}^{M}\left[T\left(Q_{n}^{(k)}+\Delta Q P_{n}^{(k)}\right)-T\left(Q_{n}^{(k)}\right)\right]^{2}}
$$




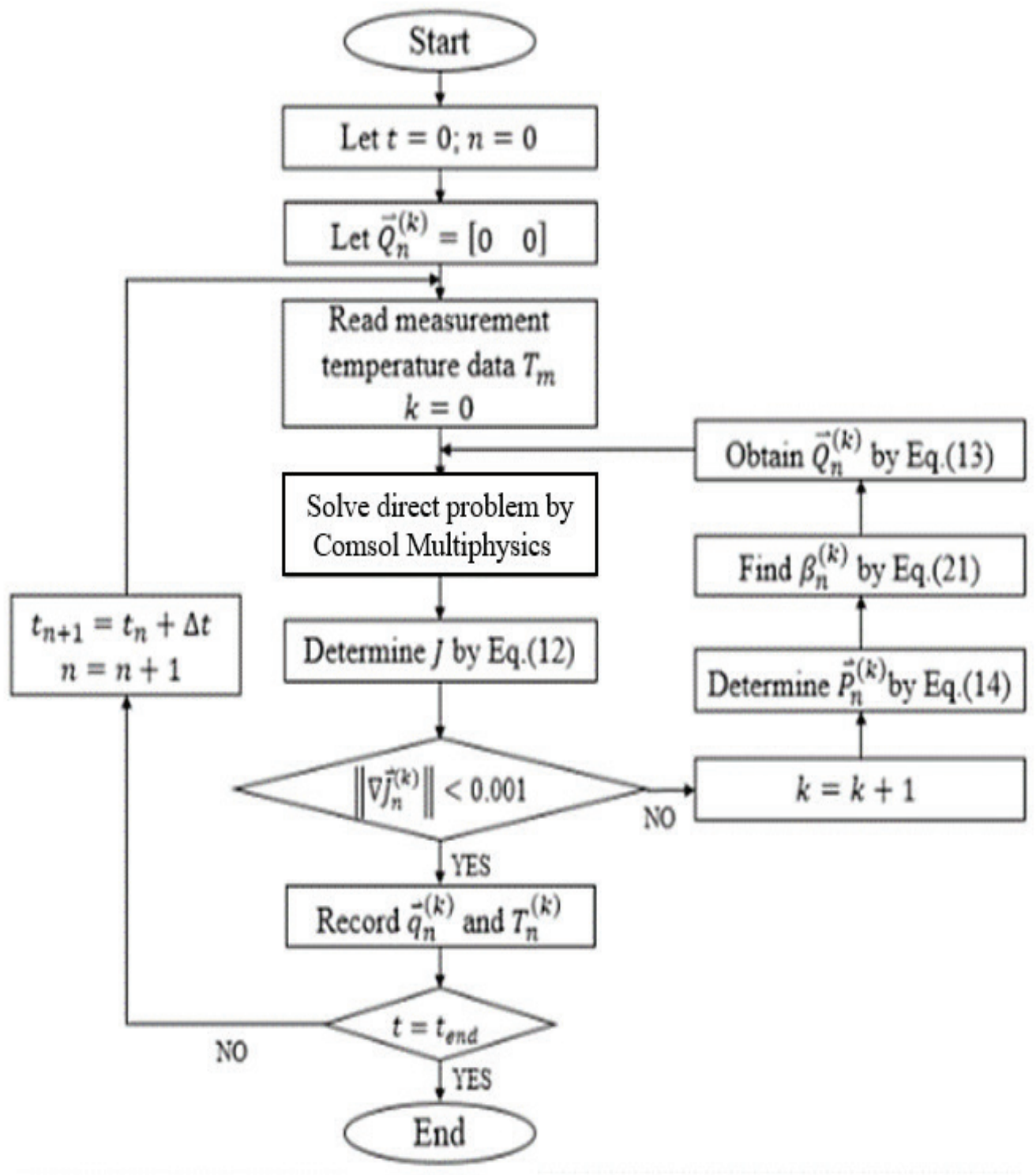

Figure 4. Flow chart of inverse method.

The conjugate direction $\vec{P}$, optimal step $\beta$ and the aforesaid parameters are substituted in Figure 4 algorithm flowchart to predict the magnitude of heat source in the front and rear bearings of spindle at any time.

\section{RESULTS AND DISCUSSION}

\section{Influence of Measurement Error, Number of Measuring Points and Measurement Position on Estimated Results}

To consider effect of above factors, inverse algorithm is implemented with using simulated measured temperature. Bearing heat source magnitude is assigned as a given value, and then the direct problem is solved. At 
the beginning, the heat source at front and rear bearing is sequentially $100 \mathrm{~W}$ and $180 \mathrm{~W}$; after that, two heat sources are reduced to $50 \mathrm{~W}$ and $90 \mathrm{~W}$ for front and rear bearings, respectively. Obtained temperature results are considered as simulated measured temperatures and used for iterative process. A standard deviation is added to simulated measured temperature by the following equation:

$\varpi=\frac{1}{\sigma \sqrt{2 \pi}} \int_{-\infty}^{T_{\varpi}} e^{-0.5\left[\left(T_{\varpi}-T_{\text {exact }}\right) / \sigma\right]^{2}}$

where $\varpi$ is total occurrence probability of the error within $0 \sim 1$, and $T_{\text {exact }}$ is the temperature simulation data without measurement error.

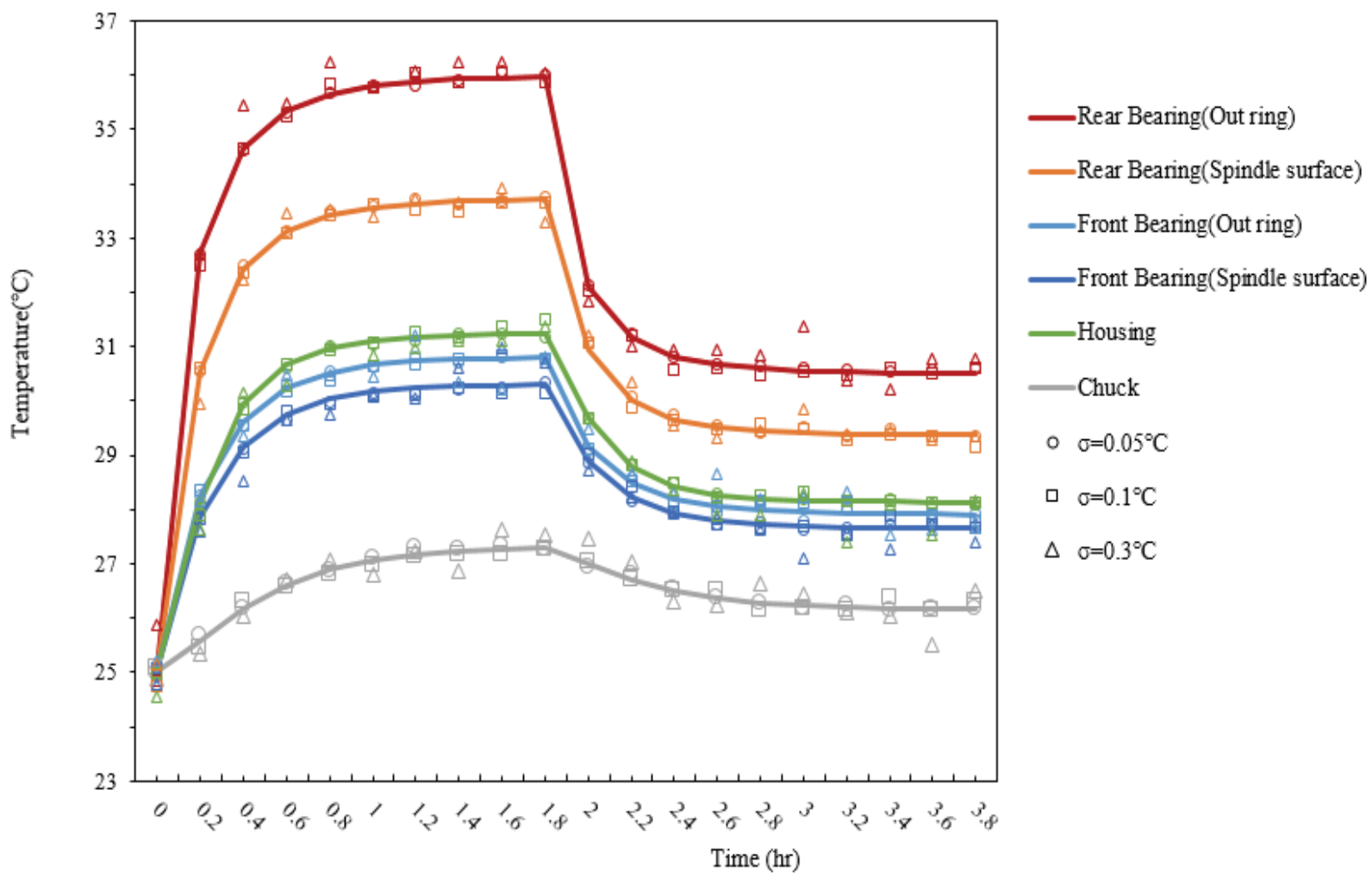

Figure 5. Temperature results under different measurement errors.

Based on these temperature data, inverse results of heat sources and temperatures with different normal distribution measurement errors are obtained and displayed in Figures 5 and 6 . Results reveal that the errors of predicted heat sources increase with increasing magnitude of $\sigma$. In addition, there are tiny different between estimated and exact temperatures. Therefore, it can be seen that the measurement error has insignificant effect on inverse results. 


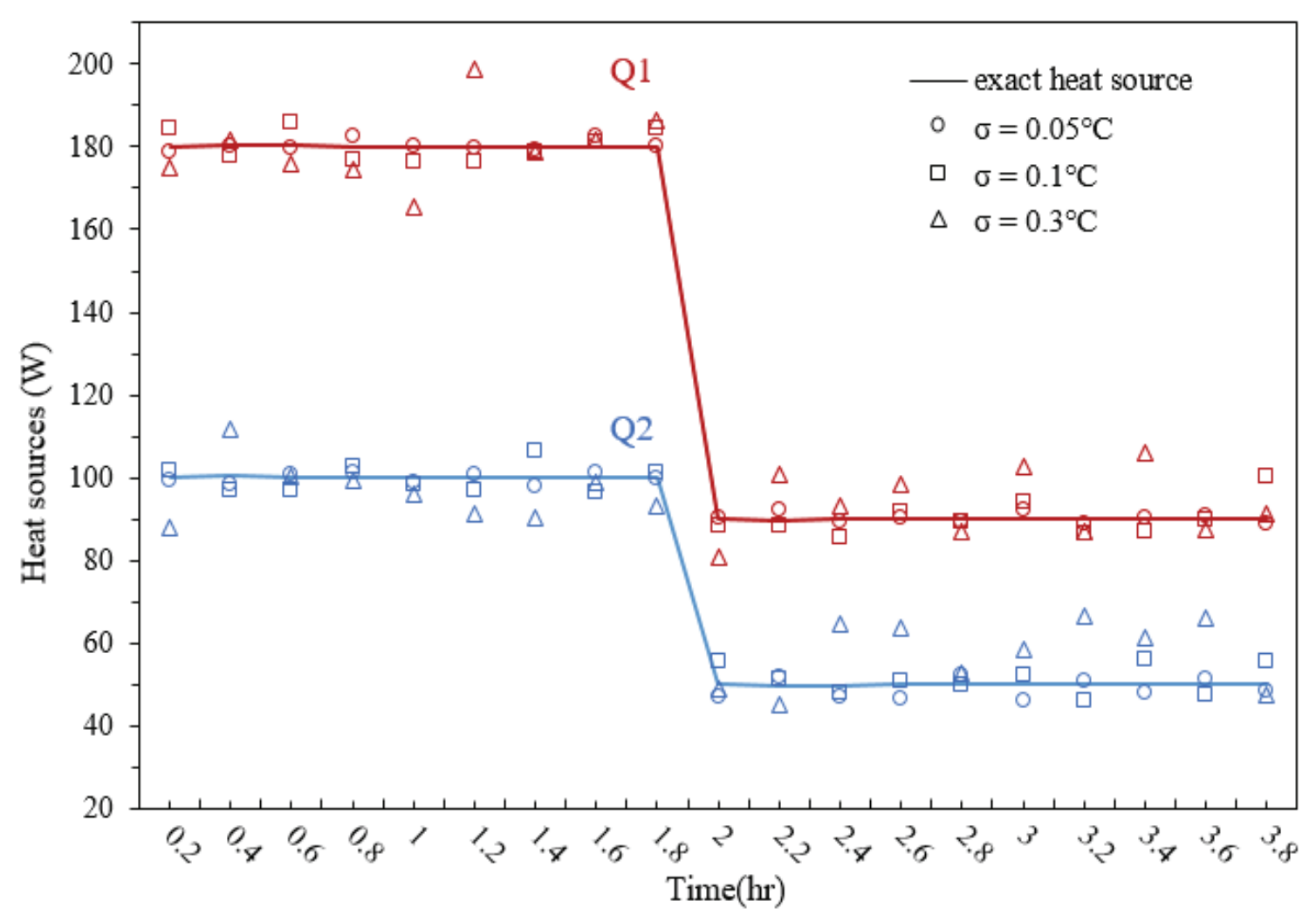

Figure 6. Heat sources results with different measured errors.

To consider the influence of the number measured points, the present inverse algorithm will be implemented with using two (at outer rings of front and rear bearings), three (at chuck and outer rings of front and rear bearings), and six (all points) temperatures. Figure 7 shows the exact and estimated heat sources of these cases. Results indicate that errors of estimated heat sources of case using three and six temperature data are not significantly improved comparing to case of two temperatures. It is because the temperature at bearing outer rings close to the heat sources; therefore, temperature at bearing outer rings are more sensitivity due to change of heat sources. Hence, these temperatures strongly provide information for inverse computation. Even if temperature at chuck and sleeve, which is far from the heat sources, is added, it has little difference of results accuracy. From above analysis, it is indicated that the heat sources can be precisely predicted based on only two measured temperatures.

Besides the effect of the number of measured points, measurement distance may influence the inverse heat source results. Different couple measurement locations are used as input data for estimating the heat sources. A comparison of exact and predicted heat sources obtained by using three couple temperatures is illustrated in Figure 8. It is noted that the measurement standard deviation herein equals $\sigma=0.1$. Results reveal that the estimated heat sources are in good agreement with exact value for cases of using measured points at bearing out rings and spindle surface. However, when using points at housing and chuck, which are more far from heat source, the inverse heat source only match with given heat source for rear bearing (95.62\%); and result for front bearing takes just $38.30 \%$ compared to exact magnitude. From these results, it can say that the measurement location has greatly influenced the accuracy of estimated results. This factor should be paid an attention during applying the inverse method for real machine in next section. 


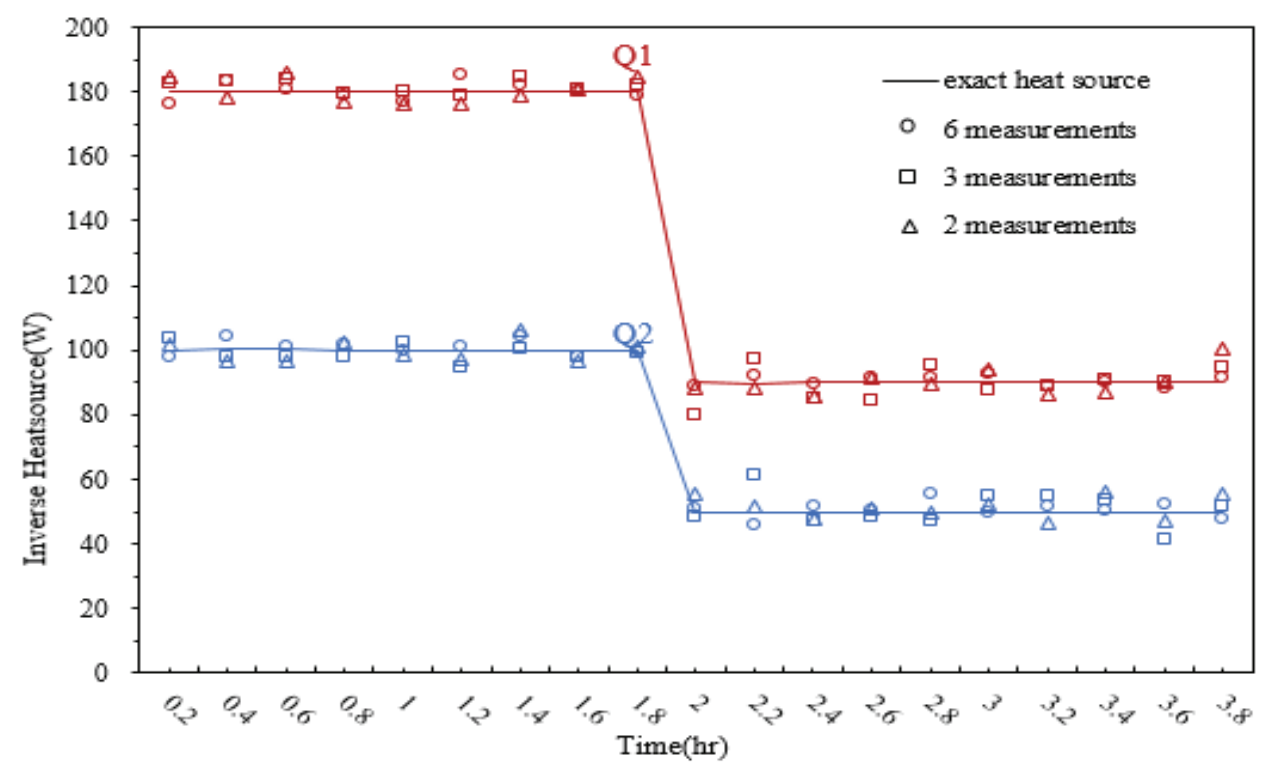

Figure 7. Comparison of estimated hear sources for different number of measured points.

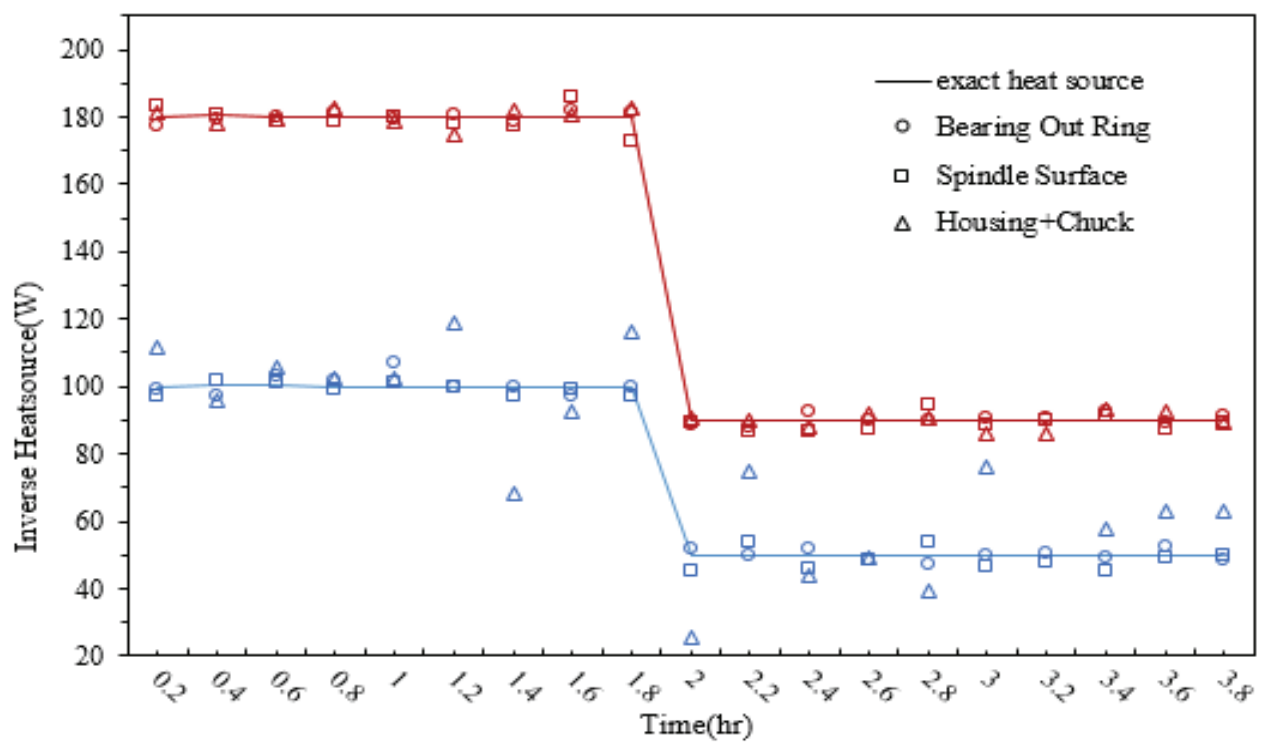

Figure 8. Effect of measurement distance on inverse results.

\subsection{Inverse Result Based on Experiential Temperature Data}

In previous section, the heat source has been successfully estimated by inverse method based on simulated measured temperatures; and temperatures at outer rings of front and rear bearings should be used in the inverse process. Firstly, the spindle is run under constant speed of $4000 \mathrm{rpm}$ for about 5.8 hours to get temperature at all points as shown in Figure 9. The temperatures at front and rear outer bearing are then extracted for input data in inverse algorithm. 


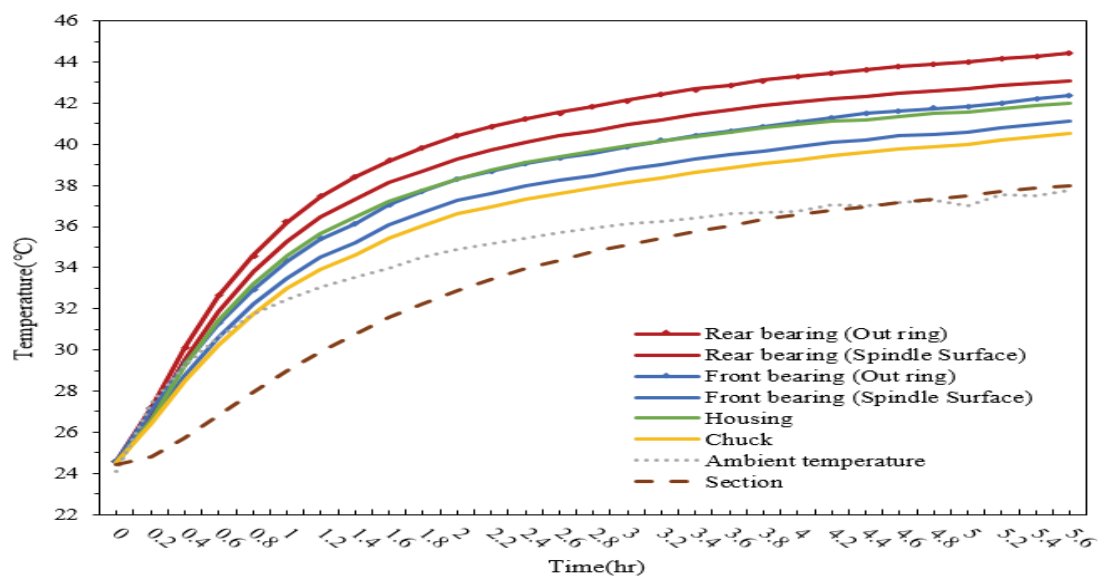

Figure 9. Time- dependent temperature under constant speed of $4000 \mathrm{rpm}$.
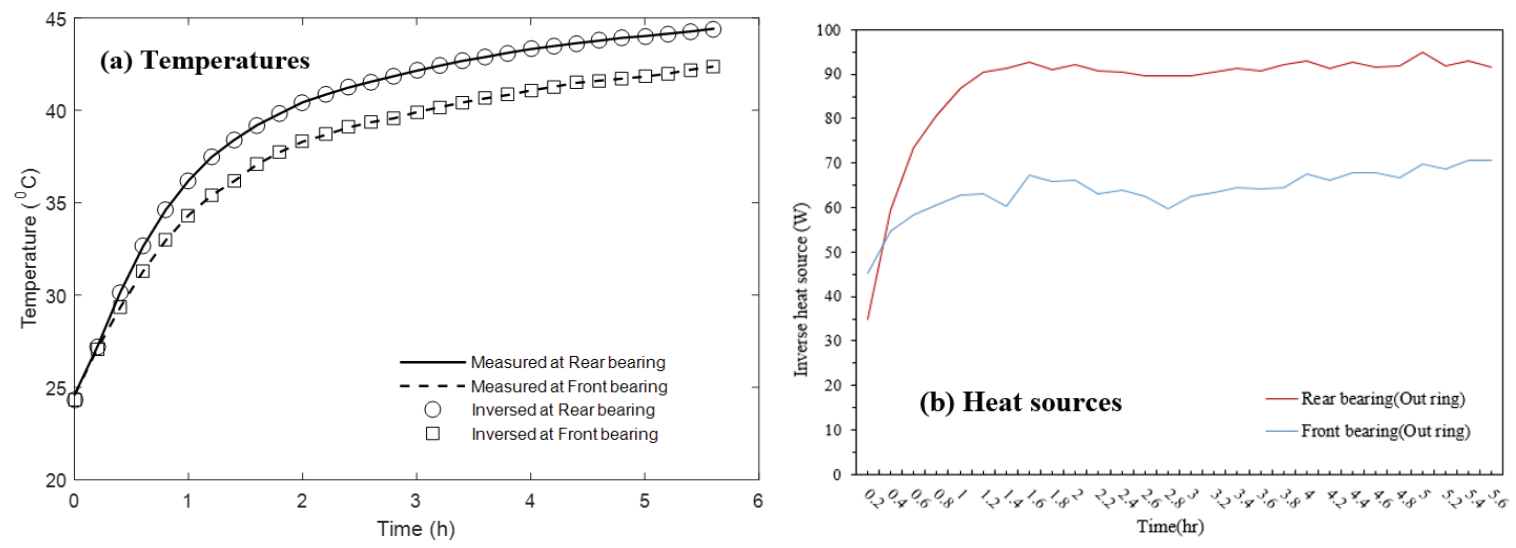

Figure 10. Estimated results for constant speed of $4000 \mathrm{rpm}$.

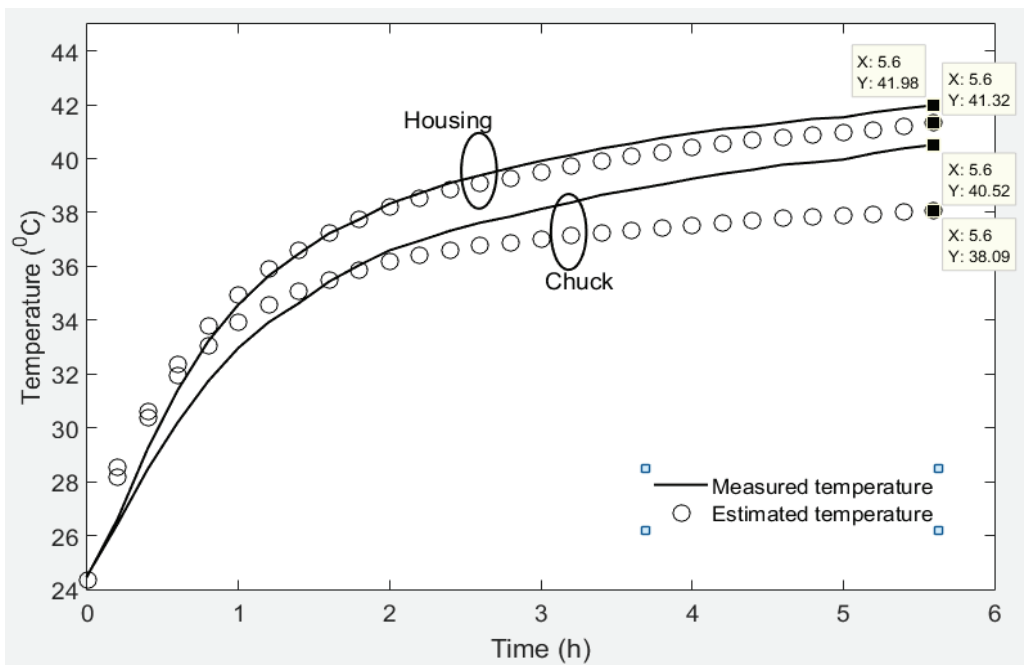

Figure 11. Comparison of temperatures at housing and chuck under 4000rpm. 
Figure 10 displays the estimated temperatures and heat sources. Results reveal that estimated temperatures are in good agreement with measured temperatures. Moreover, two other temperatures at housing and chuck are employed to validate inverse results. Consistency between estimated and measured temperature is displayed (Figure 11). Differences between estimated and experimental temperatures are $0.66{ }^{\circ} \mathrm{C}$ and $2.43^{\circ} \mathrm{C}$ at housing and chuck, respectively. Therefore, it demonstrates that the estimated results are reliable.

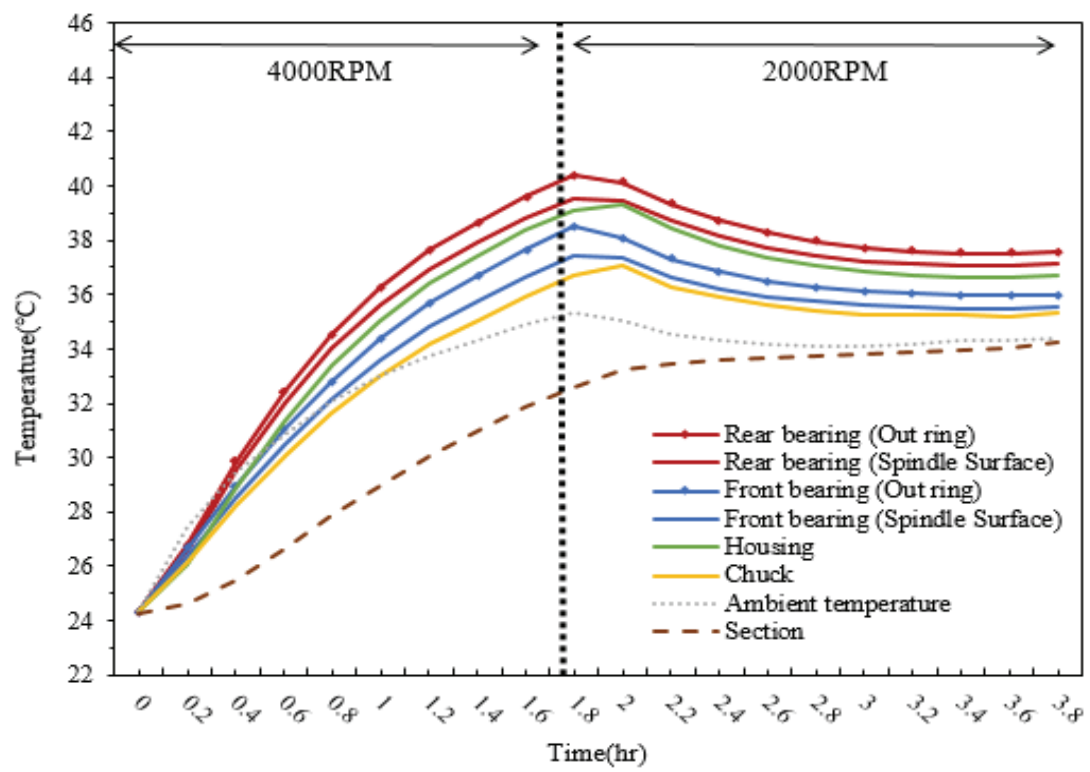

Figure 12. Experimental temperature data for varying spindle speed.
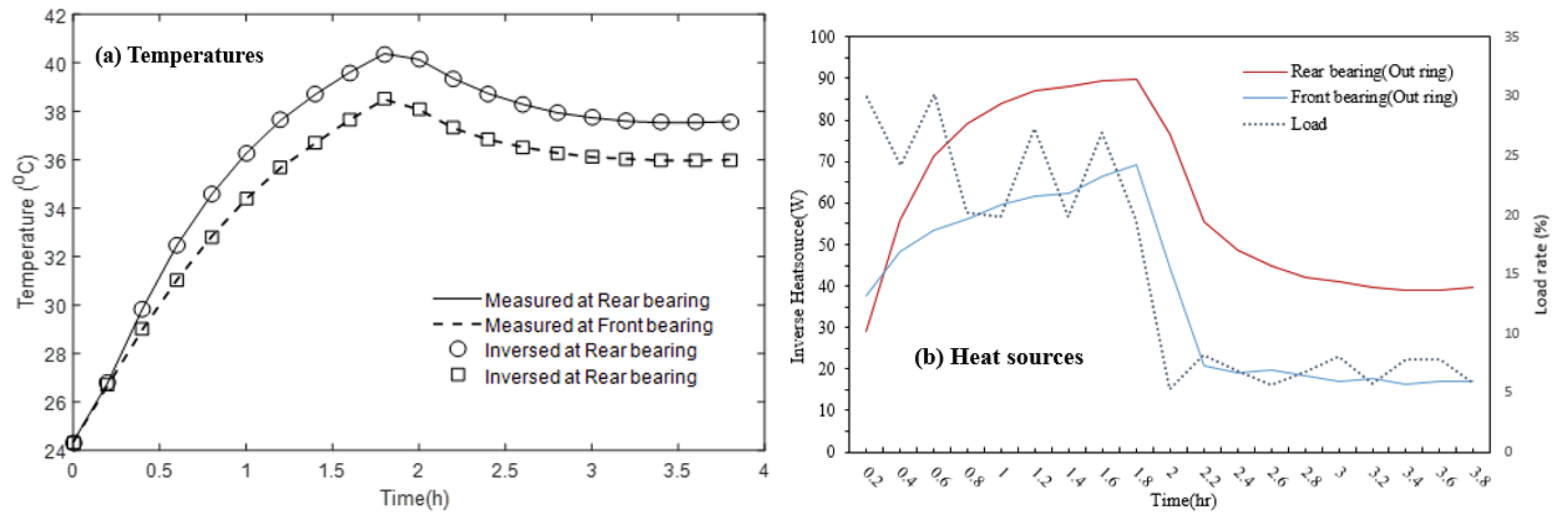

Figure 13. Estimated results for varying spindle speed case.

The inverse method is continuously applied for case of varying speed. The spindle will be run at 4000rpm (two hours) and then reduced to 2000rpm (two hours). Figure 12 presents experimental temperature recorded by all thermocouples. Predicted temperatures and heat sources are displayed in Figure 13. Again, two estimated temperatures agree well with experimental temperatures. The estimated heat sources at front and rear bearings are compared to load rate measured during the test. It can be seen that the trend of inversed heat sources is consistent with the measured load rate. In addition, the predicted temperatures also agree with experimental temperatures at 
housing and chuck as given in Figure 14. Hence, this demonstrates that the heat sources and temperatures on the lathe spindle can be accuracy estimated by current inverse method for both constant and varying speeds.

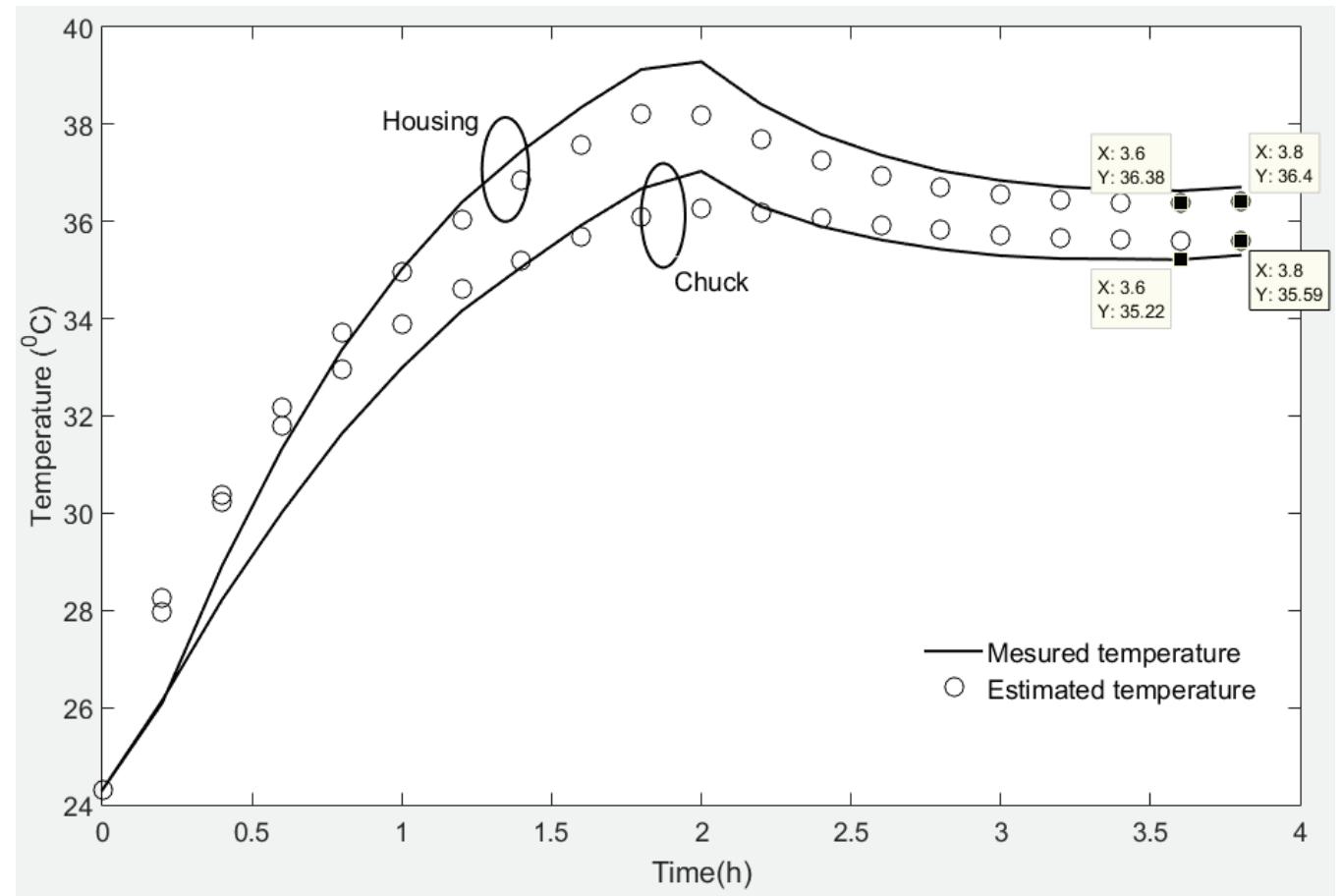

Figure 14. Comparison of temperatures at housing and chuck under varying speed.

\section{CONCLUSION}

Heat sources of spindle in horizontal lathe machine tool, which is difficult to directly measure, are successfully predicted by using inverse method based on only two measured temperatures. For simulated case, results reveal that standard errors and measurement locations have significantly influence on accuracy of the predicted heat source; the accuracy of estimated results decreases with increasing the standard errors or measurement distances. Number of measurement points has tiny effect on the estimated results. Based on experimental data, time-varying heat source at front and rear bearings is carried out for both of constant and varying speeds. Results indicate that estimated temperatures are in good agreement with experimental temperatures. A consistent trend of the predicted heat source and measured load rate is exposed. Besides, the temperature at two points on housing and chuck is also utilized to validate the estimated results. Herein, the results may provide some information to develop a real-time spindle heat source monitoring system for analyzing the bearing state and then give early warning system to prevent damage of spindle as well as machine.

\section{ACKNOWLEDGMENT}

Thanks are due to the subsidy of the Outlay MOST 107-2221-E-035-047- given by Ministry of Science and Technology, Taiwan, ROC, to help finish this special research successfully. And, thanks are due to Victor-Taichung for the machine tools and technical assistance. 


\section{REFERENCES}

Beck, J.V., 1970. Nonlinear estimation applied to the nonlinear inverse heat conduction problem. International Journal of heat and mass transfer 13, 703-716.

Bossmanns, B., Tu, J.F., 1999. A thermal model for high speed motorized spindles. International Journal of Machine Tools and Manufacture 39 1345-1366.

Childs, P., Long, C., 1996. A review of forced convective heat transfer in stationary and rotating annuli. Proceedings of the Institution of Mechanical Engineers, Part C: Journal of Mechanical Engineering Science 210, 123-134.

Churchill, S.W., Chu, H.H., 1975. Correlating equations for laminar and turbulent free convection from a horizontal cylinder. International journal of heat and mass transfer 18, 1049-1053.

Holkup, T., b, H.C., ř, P.K., Altintas, Y., Zeleny', J., 2010. Thermo-mechanical model of spindles. CIRP Annals $59,365-368$.

Huang, C.-H., Lo, H.-C., 2006. A three-dimensional inverse problem in estimating the internal heat flux of housing for high speed motors. Applied thermal engineering 26, 1515-1529.

Jedrzejewski, J., Kwasny, W., 2010. Modelling of angular contact ball bearings and axial displacements for highspeed spindles. CIRP annals 59, 377-382.

Kendoush, A.A., 1996. An approximate solution of the convective heat transfer from an isothermal rotating cylinder. International journal of heat and fluid flow 17, 439-441.

Lequien, P., Poulachon, G., Outeiro, J., Rech, J., 2018. Hybrid experimental/modelling methodology for identifying the convective heat transfer coefficient in cryogenic assisted machining. Applied Thermal Engineering 128, 500-507.

Shumakov, N., 1957. A method for the experimental study of the process of heating a solid body. Soviet PhysicsTechnical Physics 2, 771-781.

Stolz Jr, G., 1960. Numerical solutions to an inverse problem of heat conduction for simple shapes. ASME J. Heat Transfer 82, 20-25.

Wang, W.-Z., Hu, L., Zhang, S.-G., Kong, L.-J., 2014. Modeling high-speed angular contact ball bearing under the combined radial, axial and moment loads. Proceedings of the Institution of Mechanical Engineers, Part C: Journal of Mechanical Engineering Science 228, 852-864.

Yang, A.-S., Yu, X.-H., Zhuang, J.-R., Lee, C.-Y., Hsieh, W.-H., 2018. DOE-FEM based design improvement to minimize thermal errors of a high speed spindle system. Thermal Science and Engineering Progress 8, $525-536$. 